\title{
Mining Semantic Structures from Syntactic Structures in Free Text Documents
}

\author{
Hamid Mousavi \\ CSD, UCLA \\ hmousavi@cs.ucla.edu
}

\author{
Deirdre Kerr \\ CRESST, UCLA \\ dkerr@cse.ucla.edu
}

\author{
Markus Iseli \\ CRESST, UCLA \\ iseli@cse.ucla.edu
}

\author{
Carlo Zaniolo \\ CSD, UCLA \\ zaniolo@cs.ucla.edu
}

\begin{abstract}
The Web has made possible many advanced textmining applications, such as news summarization, essay grading, question answering, and semantic search. For many of such applications, statistical text-mining techniques are ineffective since they do not utilize the morphological structure of the text. Thus, many approaches use NLP-based techniques, that parse the text and use patterns to mine and analyze the parse trees which are often unnecessarily complex. Therefore, we propose a weighted-graph representation of text, called TextGraphs, which captures the grammatical and semantic relations between words and terms in the text. TextGraphs are generated using a new text mining framework which is the main focus of this paper. Our framework, SemScape, uses a statistical parser to generate few of the most probable parse trees for each sentence and employs a novel two-step pattern-based technique to extract from parse trees candidate terms and their grammatical relations. Moreover, SemScape resolves coreferences by a novel technique, generates domain-specific TextGraphs by consulting ontologies, and provides a SPARQL-like query language and an optimized engine for semantically querying and mining TextGraphs.
\end{abstract}

\section{INTRODUCTION}

The huge and fast-rising volume of free text-information now available on the Web implies that automatic summarization of textual documents is extremely desirable. Nowadays, people seek short summaries of long articles or news to find important topics in the news, and hot topics in social blogs. More importantly, structured summaries, such as Wikipedia Infoboxes, are becoming a popular feature in (semi)curated document corpora, enabling powerful structured searches [10], [17], and supporting question answering systems, and automatic personal assistants (e.g., Siri and Google Now) are getting more popular than traditional keyword-based searches.

Current text mining approaches can be divided into two main categories: bag-of-words (a.k.a statistical or machine learning) and NLP-based techniques. Since the first approaches do not exploit morphological structures in the text, they are rather ineffective and usually need to use larger data sets and ignoring less frequently mentioned information. On the other hand, NLP-based approaches parse the sentences in the text and convert them into parse trees. Parse trees contain [some of] morphological structures in the text in a more machine readable format, and thus provide a better structure for text analyzing. Although NLP-based approaches are much more resource-demanding than keyword-based ones, they are proven to be more effective in addressing the current text mining needs, specially once combined with statistical and machine learning techniques [27][28][30]. Moreover, recent advances in distributed computing techniques has hugely alleviated the time performance issue of NLP-based techniques [2].

Text mining through NLP-based techniques is often performed by employing some patterns on parse trees [22], or similar structures [25]. Generating these patterns, either manually or by statistical patten learning techniques, is a challenging and costly task. Indeed, since a simple piece of information can be expressed in many different ways through natural languages, many patterns need to be created to extract it. Generating such patterns is both costly and time-consuming, due to the fact that parse trees still carry various syntactical structures from the text. Thus, to mine parse trees one needs to provide patterns for each different structure. Therefore, a more standard form of representing the semantics of text is desirable [37], and in this paper we present a new and more expressive text representation, called TextGraph, that is much closer to the semantics of the text, and thus requires simpler and fewer patterns to be analyzed. As opposed to parse trees [22] and dependency trees [25], TextGraphs capture single- and multiword (candidate) terms and grammatical connections between words and terms in the text. Moreover, by providing weights for the edges/links, TextGraph can better handle noise in text or parse trees and the ambiguity in text.

In this paper, we present a new text mining framework, called SemScape, which employs a pattern-based technique to generate TextGraphs from free text. SemScape uses statistical parsers [22], [8] to generate few most probable pares trees of the sentences, and then uses a small set of tree-based patterns to annotate the parse trees with some useful information called MainParts. MainParts carry up the hidden information in the leaves and lower branches of the parse trees to the upper non-terminal nodes. In this way, simpler and more general patterns can be used to mine the annotated trees, referred to as the MainPart Trees (MPTs). Finally, SemScape uses another set of tree-based patterns over the MPTs to extract grammatical relations between words and terms in the text to generate the TextGraphs. Generating TextGraphs from parse trees in above two steps significantly reduces the number and the complexity of the patterns required. Therefore, SemScape makes the following contributions:

- We introduce an annotated parse tree called MainPart tree or MPT in which non-terminal nodes are annotated with important information from their branches. We also introduce a tree-based query language, called Tree-Domain (TD) rules. TD rules can be used to query both parse trees 


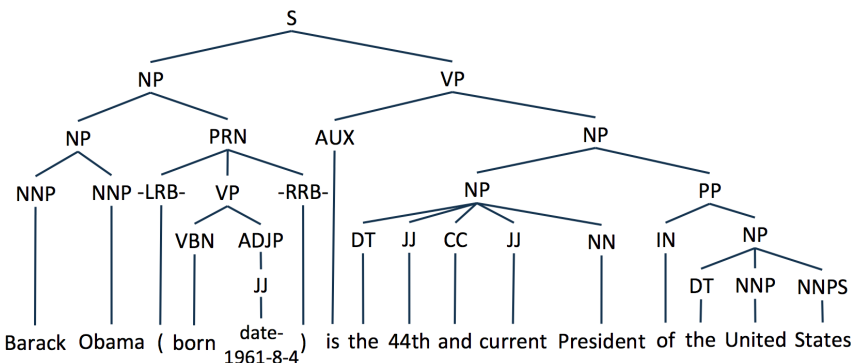

Fig. 1. Most probable parse tree for our running example.

and MPTs. Using MPTs, one can extract information from the parse trees with fewer and less complex patterns.

- Utilizing the MainPart trees, we propose a weighted graph representation of the text, called TextGraph, which hides many syntactical features of the text. TextGraphs are more expressive than tree-based structures, include multi-word (candidate) terms in the text, and are able to present ambiguity of the text through weighted edges.

- To query and mine TextGraphs, SemScape provides a SPARQL-like query language, called Graph-Domain $(G D)$. It also presents an optimization for searching graph patterns in TextGraphs which avoids several unnecessary join operations needed SPARQL engines.

- We propose a new Coreference Resolution technique through a component called Story Context (SC) which uses a large body of contextual, taxonomical, and categorical information. SC also takes advantage of many syntactical patterns specifying possible or impossible resolutions. Impossible resolutions were overlooked by previous authors, but they can the final result significantly.

- SemScape is also able to adapt with different domains by accepting an ontology. Once an ontology is fed to the framework, it generates TextGraphs with higher focus on the known terms and concept and eliminates many unrelated terms. This makes the framework capable of dealing with very noisy text data sets as shown in [30].

Since SemScape uses a pattern-based mining technique (with supports for syntactical exceptions in natural languages) to generate TextGraphs, it provides a natural way for incrementally improving the system by adding more rules to capture missing grammatical connection and exclude wrongly generated connections. Currently, all patterns mentioned in this work are created manually, however supervised or semisupervised techniques can be used to create more of such patterns. The SemScape framework has been used in several text mining applications [20][27][28][29][30], where it has proven very effective.

\section{Preparing Parse Trees}

To illustrate this and other steps toward generating TextGraphs, we use the following example text in the paper:

Motivating Example: "Barack Obama (born August 4, 1961) is the 44th and current President of the United States. He is the first African American to hold the office. Born in Honolulu, Hawaii, Pres. Obama is a graduate of Columbia University and Harvard Law School, where he was president of the Harvard Law Review."

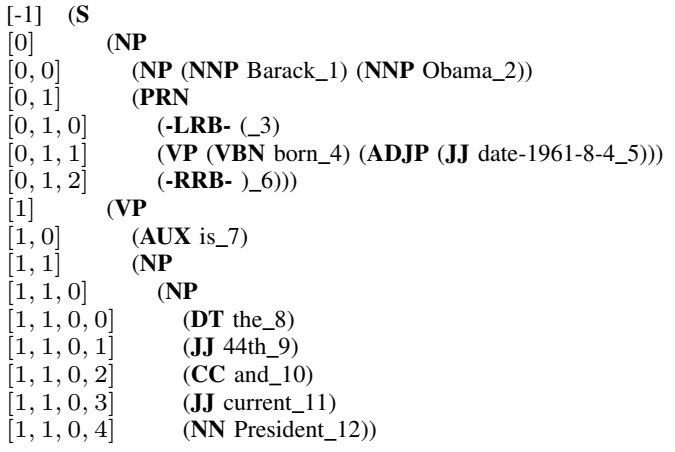

Fig. 2. Parse tree of Figure 1 in parenthesized format.

After tagging known data formats such as dates, floating point numbers, etc, and partitioning the text into its paragraphs and sentences, SemScape parses each sentence using a probabilistic parser (e.g. Charniak [8] and Stanford [22] parsers). For each sentence, we generate $N_{p t}(>1)$ parse trees $(P T \mathrm{~s})$. Having more than one PT will i) help us better deal with the inaccuracy and noisiness of the parsers in many cases, ii) increase the amount of extracted information, and iii) provide a better way for representing ambiguity in the text. For many cases, the first PT is not completely correct, so using the secondary PTs may help improving the results. Using multiple PTs also helps generating more information and capturing possible ambiguity in text. A possible PT for our example is shown in Figure 1.

Each word in the PTs is assigned an ID to make the system capable of uniquely addressing words in the text. This is required to avoid confusion among repeated words and more importantly, to preserve the order of words and terms in the TextGraphs. SemScape also uses a simple addressing scheme to address nodes in the tree (Figure 2). In this scheme, each node address contains its parent address plus its position in the ordered list of siblings.

\section{MAinPart TREeS}

Parse trees (PTs) are much richer semantic structures than the bag-of-words representation. However, they still suffer from two important issues. The most important issue is that the structure of the PTs hugely depends on the grammar and morphological structures in text. Thus they are still far from the semantics of the sentences. The second issue is that PTs (as well as dependency trees) are only connecting words together. Multi-word terms (A.k.a. Candidate Terms) and their roles in the sentences are completely missing from these structures.

To address these issues, we propose a richer structure by annotating the non-terminal nodes in the PTs with useful information about their underlying sub-trees as shown in Figure 3. For instance, consider the NP node at address $[0,0]$ in Figure 3, which is representing either 'Barack Obama' or 'Obama'. Once these pieces of information, referred to as Main Parts (MPs), will carried up to the upper nodes in the PTs, other applications will not need to search deep in the trees branches. MPs may contain multi-word (candidate) terms as well, which addresses the second issue mentioned earlier. The annotated parse trees are referred to as MainPart Trees or MPTs. To extract MPs in the PTs and assign them to their corresponding nodes, we use tree-based patterns/rules, which 


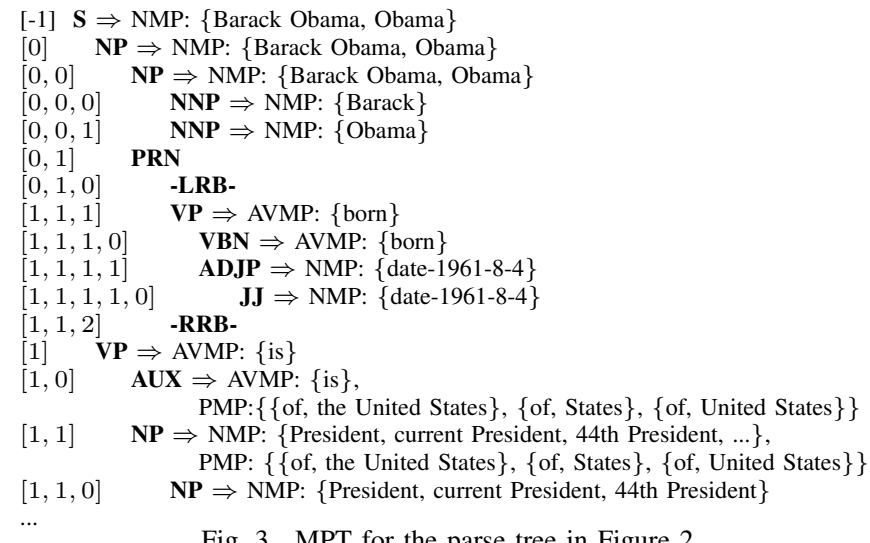

Fig. 3. MPT for the parse tree in Figure 2.

are also called Tree Domain (TD) rules. A TD rule for the mentioned MP is shown below:

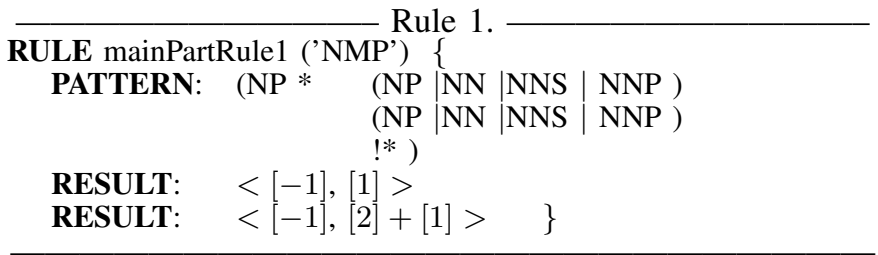

This rule consists of two parts: PATTERN and RESULT. PATTERN specifies a tree-like pattern for which we need to find matches in the PTs of the sentences in the text. The RESULT parts indicate how the MPs should be generated and to which node they should be assigned. We should add that PATTERNs are nested patterns and more expressive than regular expressions (or finite automata) [9]. This differentiates our work from most existing NLP-based techniques.

In Rule 1, PATTERN specifies noun phrases whose last two branches are both a noun phrase (NP $|\mathrm{NN}| \mathrm{NNS} \mid \mathrm{NNP})$. From the PT in Figure 1 (and in parenthesized format in Figure 2), it is easy to see that 'Barack Obama' in our motivating example matches this pattern. If any match is found for this PATTERN, the first RESULT in Rule 1 adds the NMPs of the last branch ('Obama' with address [1] in the pattern tree and address $[0,0,1]$ in the matching tree) of the matching tree to the noun MP list of its root (the node with address [-1] in the pattern tree and address $[0,0]$ in the matching tree). With its second RESULT, Rule 1 also suggests a multi-word terms, 'Barack Obama'. This sort of terms are usually referred to as Candidate Terms in the literature, and can be directly used in Name Entity Recognition systems [31]. The MPT for our running example is depicted in Figure 3 in parenthesized format. Currently, SemScape uses 135 TD rules (accessible at [5]) to generated the four types of MP listed next. Applying these TD rules over the PTs does not significantly increase the delay of generating MPTs comparing with the delay of generating PTs.

Noun MainParts (NMPs): As already explained, NMPs are defined for noun-related nodes $(N P, N N, J J, A D J P, \ldots)$, and they indicate the actual term(s) represented by these node.

Active Verb MainParts (AVMPs): A similar concept is used for the verbs-related non-terminal nodes $(V P, V B, V B Z$, $V B D, V B N, \ldots)$ in the PTs; however, since verbs have two forms, passive and active, we use two types of main-parts for verb-related nodes. Thus, AVMPs capture the active verbs of

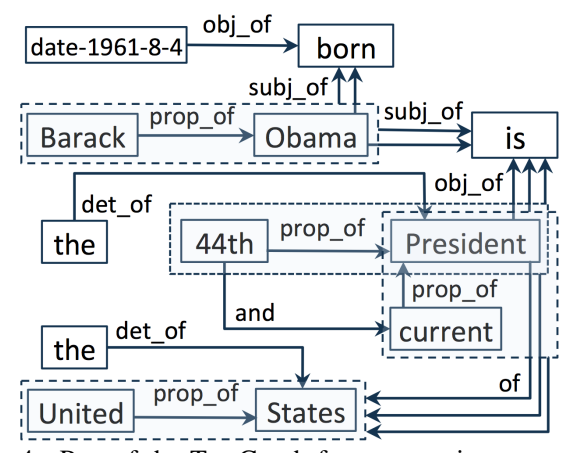

Fig. 4. Part of the TextGraph for our running example.

the verb-related nodes.

Passive Verb MainParts (PVMPs): Similar to the previous case, PVMPs are for passive verbs which are of particular importance since they change the regular roles of the subject(s) and the object(s) in the sentences.

Preposition MainParts (PMPs): The fourth MainPart set is for prepositions and preposition phrases. Both noun-related and verb-related nodes may contain PMPs. PMP of a node specifies a possible preposition for that node.

\section{TEXTGRAPHS}

MPTs provide a better structure than parse trees, but they still inherit the issues of tree-based structures mentioned earlier. In this section, we introduce a richer structured representation of text called TextGraph. TextGraphs are machinefriendly weighted graph structures, that represent grammatical connections between words and terms in the sentences, where terms are single- or multi-word phrases representing an entity or a concept. Each link in the TextGraphs is assigned a confidence value (weight) indicating SemScape's confidence on the correctness of the link and an evidence count indicating the frequency of observing the link.

A simplified TextGraph for the first sentence in our running example is shown in Figure 4. The complete list of link types in TextGraphs with their purposes is published in [30]. The graph also identifies multi-word (candidate) terms (as shown in dashed boxes) and their links to other component of the sentences. For instance, the TextGraph contains two possible subjects for the verb 'is' in sentence which are 'Barack Obama' and 'Obama'. Providing candidate terms in TextGraphs significantly facilitates the process of most text mining applications. Representing the text with graph structures makes it possible to utilize many existing graph-based mining algorithms. They are also closer to the semantics of the text, by providing meaningful terms, their grammatical relations and confidence weight for the relations. Due to the above reasons, which hugely differentiate TextGraphs from their counterpart representation techniques such as parse trees and dependency trees, more effective and efficient algorithms can be designed to extract knowledge from text by combining graph-based and statistical methods.

To generate the TextGraphs, we use more than 270 TD rules (available in [5]) to find grammatical relations between words and terms identified by the MPTs. These relations, referred to as either links or triples throughout this paper, are then combined into the final TextGraph structure. An examples for 
such TD rules is shown bellow, which rule aims at capturing the 'subject of' ('subj_of') links:

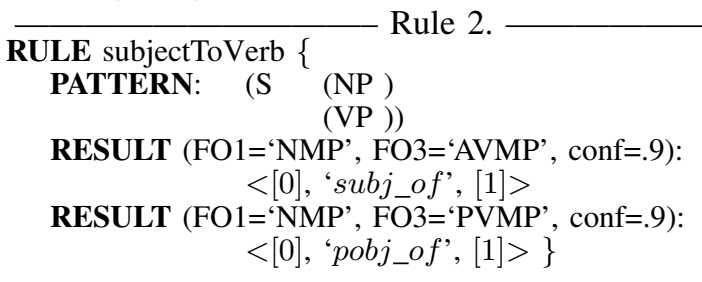

The PATTERN in Rule 2 specifies a pattern in which a Noun Phrase $(N P)$ is followed by a Verb Phrase $(V P)$. This is the most general form of subject-to-verb link structure in PTs. Similar to MP rules, Rule 2 indicates that for the matching trees, the NMPs of the noun phrase (NP) should be connected to the active verb main-part (AVMP) of the verb phrase (VP) to generate a subj_of link with confidence 0.9. Moreover, the NMPs of the noun phrase (NP) should be connected to the passive verb main-part (PVMP) of the verb phrase (VP) as a 'passive_object_of' (pobj_of) link. For our running example, this rule captures links such as <Obama, subj_of, is $>$ and $<$ Barack Obama, subj_of, is $>$.

Notice that, with the assist of MP information, this single rule can catch most of the subj_of and pobj_of links in different sentences without needing to know their lower level structures. This is actually one of the most important gains in the SemScape framework, that dramatically decreases the number and the complexity of required patterns/rules required for any text mining application.

Generated links have confidence values (indicated by keyword 'conf' in Rule 2) showing SemScape's confidence on the correctness of the link. If the same link is generated from different rules or MPTs, SemScape increases its correctness confidence as discussed in Subsection IV-B. After applying all rules to the MPTs and generating the triples, we combine them into the final TextGraph (e.g. Figure 4).

\section{A. Support for Exceptions}

Syntactic exceptions are the inseparable part of any natural languages. Although capturing exceptions can significantly enhance the quality of the text mining systems, most of existing approaches do not provide an easy-to-use technique to handle exceptions. Moreover, finding patterns with no exceptions in natural languages is a very tedious task, and a general pattern should be split down into many smaller patterns too avoid some exception cases. To simplify this process without needing to split our general patterns (e.g. Rule 2), SemScape uses patterns with negative confidence to specify exceptions and remove many of the incorrectly generated triples from the TextGraphs. Thus if the same triple is extracted multiple times by different rules over the MPTs of the same sentence, and one of the extracted triples has a negative confidence, the triples with positive confidence will be eliminated.

For instance, consider the sentence "In the woods are trees". Since the sentence is in inverted form, which is not as common as the normal form, the parsers may not be able to recognize the structure correctly. For instance for the mentioned sentence, only one PT from the first three suggested PTs by Stanford parser is correctly capturing the inverted form.
Thus, our patterns may generate incorrect information from the incorrect PTs. $(<$ trees, obj_of, are $>$ in our case $)$. To eliminate this incorrect information, we use the following pattern:

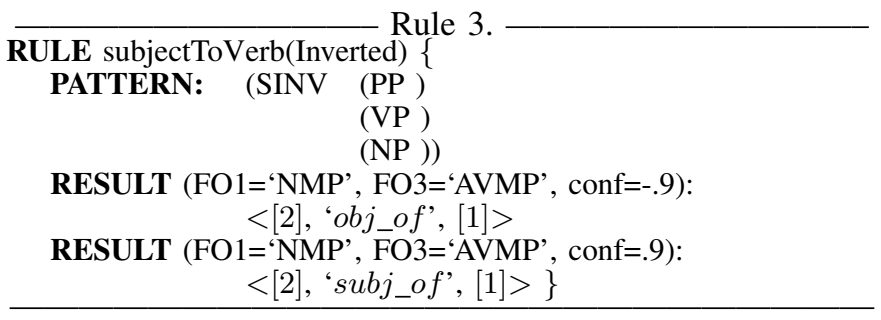

This pattern matches the correct PT and generated a triple with negative confidence $(<$ trees, obj_of, are $>)$ as well as the correct triple $(<$ trees, subj_of, are $>$ ). Using the negative triple, SemScape can eliminate incorrect triples generated from wrong PTs and improve the final TextGraph for the sentence.

\section{B. Combining Confidence Value}

Since the same triple may be generated more than once (from different rules or different PTs), we need to combine their confidence value $c$. Similar to [24], the only assumption for the combination process is that evidences of the same piece of information are independent from each other. Thus, if a piece of information has been generated twice by different rules or from different MainPart Trees, once with confidence $c_{1} \geq 0$, and once with $c_{2} \geq 0$, we combine the confidence to $c=1-\left(1-c_{1}\right)\left(1-c_{2}\right)=c_{1}+\left(1-c_{1}\right) c_{2}$. This new confidence is higher than both $c_{1}$ and $c_{2}$ which indicates the link's correctness probability is now higher. For each triple, we also count the number of time it has been generated and refer to it as the evidence frequency or count $(e)$. We should note that, if one of the confidence values is negative for a particular triple (specifying an exception), we eliminate all same triples with positive confidence as explained in previous subsection.

\section{Enriching TextGraphs with Ontologies}

Another important feature of SemScape is the ability to adapt an ontology and provide more related candidate terms to the specified ontology. The Ontology here can be both domain-specific or domain independent. For simplicity, one can also specify a list of concepts instead of Ontology (e.g., the list of all subjects in Wikipedia). This feature has two main advantages discussed next.

The first advantage is to control the volume of generated Noun MainParts. For complex noun phrases (NPs) there might be several possible candidate terms. Not all of these candidate terms are useful or meaningful. Therefore, suggesting all of them as MPs may lead to a very large set of candidate terms which lowers the efficiency of the system. To prevent this problem, SemScape is made capable of utilizing an ontology, say $O$. Using $O$, SemScape only generates candidates terms that either i) contain less than three words, ii) are part of an existing concepts in $O$, or iii) contain a concept from $O$. In most simple cases this generates all possible candidate terms; however for many long noun phrases, this helps us reduce the size of the TextGraphs. The second advantage of incorporating an ontology is to allow domain-specific applications to better utilize the framework. This can also spot more related parts 
of the text with respect to the ontology which consequently improves system's robustness on dealing with noisy corpora. This feature is discussed in greater detail in [30].

\section{Graph Domain Patterns}

SemScape provides a graph-based query language, called Graph Domain or GD rules, to let users and applications mine the TextGraph. Although the format of GD rules is very similar to that of SPARQL [6], its implementation is slightly different as explained in Section VI. To simplifying the mining process from TextGraphs, GD rules support few extended features that are introduced later in this subsection. Besides the external applications that can benefit from GD rules, SemScape uses GD rules for two purposes. The first one is to complete and improve TextGraphs using GD patterns. These patterns are often much easier to be expressed by GD rules than TD rules. The second purpose is to perform Coreference Resolution which is the topic of the next section. Readers are also referred to [5], [30] and [29] for more examples of such rules.

We should add that GD rules are usually considered as in batch of patterns aiming at mining certain types of information. That is SemScape may be fed with sets of GD rules for different tasks (and applications). For instance as shown in [30], we fed the system with rules for generating ontological links in an automatic ontology generation system. Similar idea is used in [28] to generate structured information from free text. Thus, once the GD rules are specified by an application, SemScape will apply them on all TextGraphs of the provided text, combine the resulted tuples, and report them.

Besides SPARQL's features, GD rules introduce the following features to ease the process of TextGraphs mining. Each rule may have multiple SELECT clauses to allow generating multiple pieces of information from same patterns. One may use keyword 'NEG' before the SELECT keyword to specifying exceptions similar to TD rules in subsection IV-A. One may use keyword 'NOT' before any triple in the WHERE clauses to indicate the absence of some links in the pattern, which requires a more complex expression in SPARQL.

\section{Coreference Resolution}

In textual documents, many pronouns and references are used to refer to other terms (concepts). For instance in our running example, the pronoun ' $h e$ ' in the second sentence is referring to 'Barack Obama' and 'Pres. Obama' is a reference for 'Barack Obama'. Resolving these types of coreferences, called Coreference Resolution, is a very challenging task since it requires a huge amount of contextual knowledge, commonsense knowledge, and in many cases complex and ad hoc inferencing techniques [26]. The ambiguity of the natural languages also aggravates this issue.

In SemScape, we propose a new technique to resolve coreferences through the Story Context (SC) component. At its highest level, SC recognizes characters (A.k.a. mentions) in the text, learns contextual information about them from different resources, and uses this contextual information and a novel pattern-based technique to match characters to each other and resolve coreferences in the text. These steps are explained next in this section.

\section{A. Recognizing Characters}

The first step to construct the SC's structure is to recognize possible characters or mentions. This is a relatively easy task for us since TextGraphs already provide all the candidate terms (Noun MainParts) in text. We use these candidate terms as the characters of [the story of] the text. However, some characters in the text are more important (due to their role) than the others, and as a result they are more probable to be referred with pronouns or other references. To determine the importance of a character, each character is assigned an evidence count and a confidence weight. Whenever the same character is encountered in different roles (relations), we increase its evidence count by one and its confidence as explained in Section IV-B. At this stage each occurrence of the same candidate term is considered as a separate character.

\section{B. Mining Characters Context}

After creating characters, we gather information on some of their important attributes or properties. These properties are sometimes called agreement properties. Currently, we consider seven properties: isMale (if it is a male or a female), isPerson (if it is a person or not), isOrganization (if it is an organization or not), isLocation (if it is an geographical location or not), isAnimal (if it is an animal or not), isObject (if it is a thing or not), and isPlural (if it is plural word or not). Other properties could be added to this set to improve the final resolutions results. However, we found these the most useful and differentiating properties. We should add that most of the exiting works in this area only consider person, gender, and number as their agreement properties [18][23][11][16].

For each property, SC uses different sources of information to estimate their value, which ranges between -1 and 1 . Value 1 means $100 \%$ confidence that the property holds and value -1 means $100 \%$ confidence it does not (value 0 indicates no information about the property). As opposed to most similar approaches which use only true/false values for characters properties [18][23][11][16], SC uses probability of being true or being false to better deal with uncertainty .

To evaluate the mentioned properties, we use Wikipedia's categorical information. For instance if one of the ancestor of a character is the 'Category:People' category in Wikipedia, we set its isPerson property to 1 . The same approach is used for isOrganization, isLocation, isAnimal, and isObject properties. This technique can be used for many potential properties that one may want to add to our initial list of properties. However, the main drawback of this technique is that for many characters, there is either no equivalent title in Wikipedia or no categorical information. Thus as provided in the next paragraph, we use several heuristics to mine more contextual information on each character.

We use VerbNet [21] which for each verb $v$ specifies its possible subject or object as either an organization, a person, an animal, or an object. For instance, in the sentence 'The agent was killed in a terrorist attack.', due to the meaning of the verb 'killed', 'agent' is probably a person or an animal. For isPlural, we use the POS tags generated from the parser as well as some TD rules (e.g., terms containing the word 'and' or ' $o r$ ' are considered plural). For isMale, we use lists of masculine 
(e.g., waiter, king, etc.) and feminine (e.g., waitress, queen, etc.) terms and lists of male or female proper first names. For isPerson, we use some POS tag information (e.g., NNP), our male and female first names lists, as well as some TD rules (e.g., any term renaming the words 'who' or 'whom' is a person). We also use a list of animal names to add more evidence to isAnimal. For isObject, if there is evidence that a term is not a person and is not an animal, we increase the confidence that it is an object. Any term renaming the word 'which' is also an object.

Combining Potentially Referencing Characters: Another new technique to improve our understanding about the values of the seven properties for different characters is to use taxonomical relations namely type_of relations between the characters. These relations can be generated using our OntoHarvester system [30] and Hyponym information in WordNet [36]. The key idea is that if character $\gamma_{1}$ is type_of character $\gamma_{2}$ with confidence $c$, then $\gamma_{2}$ may be used as a coreference for $\gamma_{1}$ (in other words, $\gamma_{1}$ may be referred to as $\gamma_{2}$ ) with confidence $c$. For instance, since 'algebraic equation' is type_of 'equation', after the first time 'algebraic equation' is mentioned in text, it can be referenced with the 'equation' for the rest of text. This essentially means that $\gamma_{1}$ can inherit the properties of $\gamma_{2}$ and vice versa. In order to do so, for each property $f$ of $\gamma_{1}$, we update its confidence value, $\gamma_{1} . f$, as follows:

$$
\gamma_{1} \cdot f= \begin{cases}\gamma_{1} \cdot f+\left(1-\gamma_{1} \cdot f\right) c \gamma_{2} \cdot f & \text { if } \gamma_{1} \cdot f \times \gamma_{2} \cdot f \geq 0 \\ \left(\gamma_{1} \cdot f+c \gamma_{2} \cdot f\right) / 2 & \text { if } \gamma_{1} \cdot f \times \gamma_{2} \cdot f<0\end{cases}
$$

The idea of combining the confidence values is essentially the same as in Section IV-B if properties' values have the same signs. If they have different signs, we simply take a weighted average on their values. By propagating the values of the properties for potentially coreference characters, we ease our later resolution technique which is based on the similarity (or agreement) of the properties of the characters.

\section{Finding Patterns for Coreferences}

Although in general resolving coreferences only based on morphological structures in text is not feasible, there are few cases for which these structures may indicate a resolution. For instance consider the sentence "Bob relieved himself telling him the truth.". Clearly from the structure of the sentence, one can tell the reflexive pronoun'himself' refers to ' $B o b$ ' in this sentence. Here the pattern is that if the object of a verb is a reflexive pronoun, it always refers to the subject of the same verb. To capture such a pattern, we use the following GD rule:

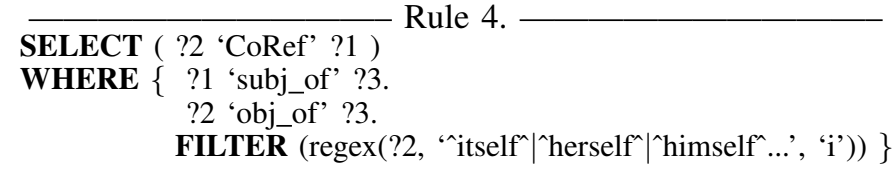

In addition to reflexive pronouns, we use predictive nominative constructs, first exploited by [33], appositive and role appositive constructs [16], and relative pronouns construct [34]. As can be seen, there are only very few such constructs that explicitly specify a resolution. However, there are many cases that a construct explicitly indicates two characters can NOT refer to each other (be each others resolution). For instance, in our earlier example, 'him' can refer to neither ' $B o b$ ' nor 'himself'. We refer to such cases as impossible resolutions. In general, the object and subject of most verbs can not be each other's resolutions unless the object is a reflexive pronoun. This pattern is specified by the following GD rule:

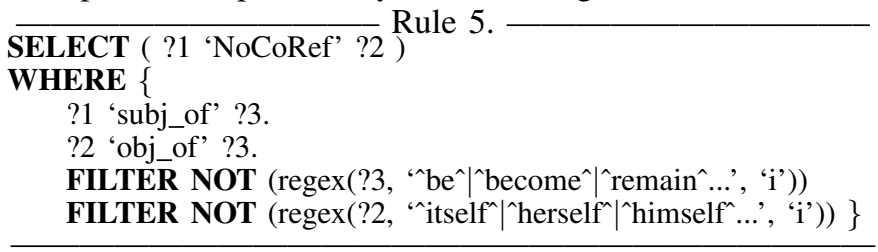

Some other obvious constructs indicating impossible resolutions are terms connected through a preposition, terms for which one is part of the other, and terms connected with conjunctions ('and', 'or', 'except', etc.) Currently, we use 64 GD rules (available at [5]) to extract possible and impossible resolutions from the morphological information in text. For possible resolutions, we combine the characters as explained in the previous subsection. The impossible resolutions, on the other hand, are used in the next subsection where we explain how SemScape performs the final character resolution.

\section{Resolving Characters}

Say that a character $\gamma_{1}$ from a sentence $s$ needs to be resolved. We calculate the similarity (see next paragraph) between $\gamma_{1}$ in $s$ and all other non-pronoun characters in $s$ that are not an impossible resolution for $\gamma_{1}$. Our studies shows that filtering by impossible resolution reduces the search space by more than half for each sentence. Similarity values larger than a predefined threshold are reported as resolutions. If no value exceeds the threshold, resolution search for $\gamma_{1}$ is continued among characters in the previous same-paragraph sentences, iteratively. In this way, we take the recency into account. If $\gamma_{1}$ is a pronoun and no resolution is found for it in its paragraph, the search will be stopped; this should be a rare case. If $\gamma_{1}$ is not a pronoun and it is not resolved yet, sentences of the previous paragraph are also checked until a resolution is found or until the beginning of the document is reached. In other words, the scope for a pronoun's resolution is only its paragraph in SemScape, while the scope for other coreferences is the entire document.

Characters Similarity Measurements: To compute the similarity of two characters $\gamma_{1}$ and $\gamma_{2}$, we define the distance for property $f$ as $\delta_{f}=\left(\gamma_{1} \cdot f-\gamma_{2} \cdot f\right) / 2\left(-1 \leq \delta_{f} \leq 1\right)$ and average for property $f$ as $\mu_{f}=\left(\gamma_{1} \cdot f+\gamma_{2} \cdot f\right) / 2\left(-1 \leq \mu_{f} \leq 1\right)$ respectively. Thus, we compute the similarity of characters $\gamma_{1}$ and $\gamma_{2}$ by $1-\left(\sum_{f \in F} d_{f}\right) /|F|$, where $F$ is the set of all properties (i.e. $|F|=7$ in our case) and $d_{f}$ is the dissimilarity of $\gamma_{1}$ and $\gamma_{2}$ for property $f$ which is defined as $d_{f}=\left|\delta_{f}\right| /\left(\left|\mu_{f}\right|+1\right)$. It is easy to see that $0 \leq d_{f} \leq 1$. The above formula indicates that more difference $\left(\left|\delta_{f}\right|\right)$ essentially means more dissimilarity, especially when the average is smaller (e.g., the dissimilarity of properties with values -.2 and .2 is more than the dissimilarity of properties with values .5 and .9 even though their distances are the same).

\section{GRAPH MATChING OPTIMIZATION}

TextGraphs can be presented in RDF format, and thus queried by SPARQL. However, there is a subtle difference 


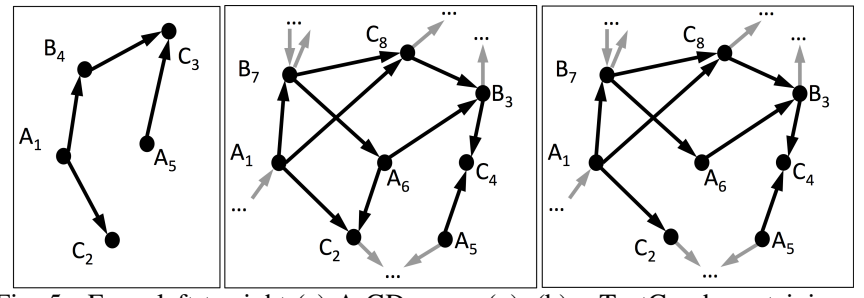

Fig. 5. From left to right (a) A GD query $(q)$, (b) a TextGraph containing $q$ $(t g)$, and (c) a TextGraph not containing $q\left(t g^{\prime}\right)$.

between TextGraphs and RDF graphs. As the TextGraphs are the graph representation of text, they may contain several nodes with the same name or label while nodes in RDF must have unique names. For instance in the TextGraph in Figure 4 , there are two nodes with label 'the'. This makes querying the TextGraphs using SPARQL inefficient as explained next.

Consider the GD pattern/query $(q)$ in Figure 5(a). Five nodes with three different labels $(\mathrm{A}, \mathrm{B}$, and $\mathrm{C})$ are connected through four links in this query. As mentioned earlier, to differentiate among nodes with the same label, SemScape assigns an ID to each node (e.g. 'the_ 8 ' and 'the_14' in Figure 4). To ease our discussions, we assume all edges have the same label, say 'link'. Now consider two TextGraphs $t g$ and $t g^{\prime}$ in Figures 4(b) and 4(c). Although both $t g$ and $t g^{\prime}$ contain all the individual edges of the query graph $q$, only $t g$ contains a subgraph matching $q$. To find such matches, $q$ can be expressed with the following SPARQL query:

\section{SELECT $*$} Rule 6.

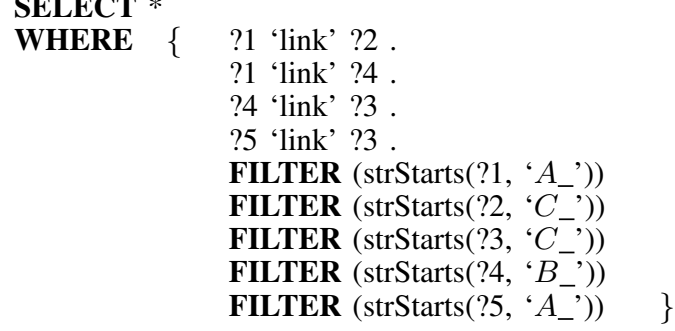

Although this approach perfectly works for GD query $q$ using any SPARQL engine, it is very inefficient and slow, since it can not take advantage of any smart indexing techniques. Notice that the engine normally needs to traverse all triples for each where clause in the form of $<? 1$ 'link' ?2 . $>$ and then filter them based on the specified FILTER commands. The same phenomenon can happen in other RDF resources, however it is much more frequent in TextGraphs due to too many same-label nodes.

To take advantage of the same-label nodes in our engine, we first ignore all the IDs assigned by SemScape to nodes and collapse both query graphs and TextGraphs by combining same-label nodes into a single node. The collapsed version of
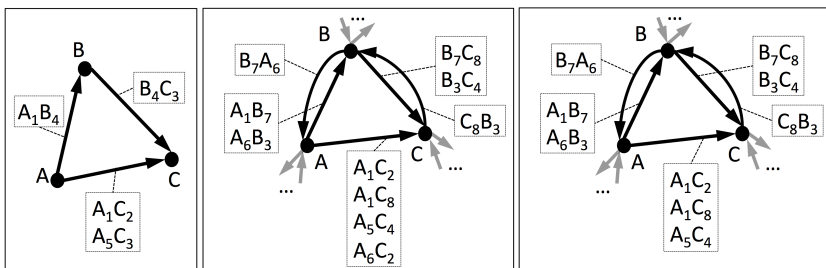

Fig. 6. From left to right: Collapsed version of (a) $q\left(q_{c}\right)$, (b) $t g\left(t g_{c}\right)$, and (c) $t g^{\prime}\left(t g_{c}^{\prime}\right)$.

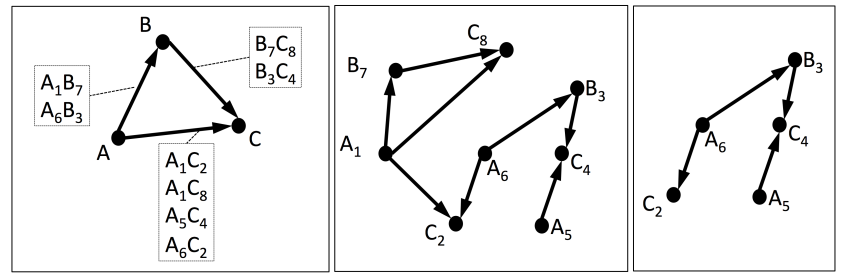

Fig. 7. From left to right: (a) the subgraph of $t g_{c}$ matching $q_{c}$, (b) the expanded graph of part a, and (c) the subgraph of $t g$ matching $q$.

the examples in Figure 5 is depicted in Figure 6. As shown in Figure 6, for every link in the collapsed version, we store all associated links in the actual graph for later retrieval. Now the collapsed version of query $q$, called $q_{c}$ (Figure 6(a)), can be expressed by the following SPARQL query:

\begin{tabular}{ll}
\hline SELECT $*$ & Rule 7. \\
WHERE \{ & 'A' 'link' 'C'. \\
& 'A' 'link' 'B'. \\
& 'B' 'link' 'C'.
\end{tabular}

Answering collapsed queries (e.g. Rule 7.) over collapsed TextGraphs is much faster than regular queries (e.g. Rule 6.) over original TextGraphs. This is because of i) the collapsed queries are smaller in the number of edges which consequently reduce the number of join operations needed by SPARQL engines, and ii) there are more literal nodes and less variable nodes in the collapsed query graphs, which makes a better use of indexing optimizations. However, finding a match for a collapsed query on a collapsed TextGraph does not necessarily mean that the actual query matches the actual TextGraphs (e.g., $q_{c}$ matches $t g_{c}^{\prime}$ but $q$ does not match $t g^{\prime}$.) A match, here, only indicates that all edges in the query graph have at least one corresponding edge in the TextGraph. This check significantly reduces the search space by eliminating many TextGraphs in which one or more of the specified edges in $q$ is missing. For the remaining cases, we use the following verification algorithm to verify that the matching edges construct the same graph structure specified by $q$.

Verification: After finding the matches for the collapsed queries on the collapsed TextGraphs, we expand the matching edges to those in the actual edges. For instance, consider TextGraph $t g$ and its collapsed version $t g_{c}$ (Figure 6(b)). Running the collapsed query $q_{c}$ over $t g_{c}$ will return the subgraph shown in Figure 7(a). Once we expand this subgraph to its corresponding subgraph in $t g$, we create a graph in which each edge matches at least one of the edges in the original query graph $q$. This subgrapg which is shown in Figure 7(b) is referred to as $t g_{m}$.

Now, the goal is to verify that the query graph $q$ matches with $t g_{m}$ or part of it. To this end, any sub-graph matching technique can be utilized. Fortunately, since both nodes and links in TextGraphs are labeled and the queries are mostly small graphs, the problem is much simpler than the general subgraph matching (subgraph isomorphism) problem which is NP-Complete [12]. In our current implementation, we use a simple recursive backtracking procedure.

\section{RELATED WORK}

Text-Mining Frameworks: Several NLP-based text analyzing packages and frameworks have been introduced in recent 
years. Apache UIMA [2], OpenNLP [1], NLTK [3], GATE [13], and CoreNLP [7] are among most commonly used such systems, which mostly support a limited set of basic NLP tasks such as POS-tagging, parsing, NER, etc. However to our knowledge, none of these systems are able to support semantic analysis and querying (such as GD patterns) of the free text. Semantic Representation of Text: Although much efforts are devoted to represent knowledge in more machine readable structures such as RDF graphs [4], there are very few research efforts to semantically represent text for the purpose of more effective and efficient text querying and mining. We already discussed the shortcomings of parse tree [22] and dependency tree [25] representations. But unfortunately most NLP-based text-mining approaches including the ones mentioned above are still using these tree-bases representations, due to lack of a better semantic representation [37]. There are some efforts to present textual knowledge in Concepts Maps [32], Concept Graphs [35], etc. However, the fact that nodes in these graphs must be unique concepts makes them less effective for text representation. Moreover, a general approach for automatically converting text into such formats is still lacking

Coreference Resolutions: Hobbs [18] introduced one of the early pronoun resolution techniques. In [19], Kehler et al. utilized shallow parsing to resolve third-person pronouns and achieved only marginal improvement with respect to Hobbs. Authors in [23] created a set of potential resolutions for each pronoun, and used recency, syntax-based preferences, and a few agreement properties to suggest the final resolutions. Authors in [14] used a similar approach by employing the centering theorem. These approaches are only able to resolve pronouns and suffer from low accuracy problem. Thus, more recent works focus on either capturing some syntactic structures implying a resolution to combine them with semantic features [33][16][34] or utilizing statistical techniques [15][11]. However, these approaches do not use taxonomical information and constructs for impossible resolutions, and can not identify the correct multi-word referent as they either consider the whole NP or single-word terms.

\section{CONCLUSION}

Mining semantic information from free-text documents will provide the enabling technology for a host of important new applications. NLP-techniques, which utilize the morphological structure of the text to produce plausible parse trees, represent an important first step that must be followed by anaphora resolution, and consultation of contextual and taxonomical information from knowledge bases, including domain-specific ontologies. To perform these tasks, we have proposed a TextGraph representation and several TextGraph based techniques. These proved quite effective at identifying the semantic relationships between entities, properties and attributes in the sentences, and between those and their realworld counterparts. A number of experiments, including our recent text-mining of the whole Wikipedia [28][29] illustrate this conclusion.

\section{REFERENCES}

[1] Apache opennlp. https://opennlp.apache.org/.

[2] Apache uima. http://uima.apache.org/.
[3] Natural language toolkit. http://www.nltk.org/.

[4] Resource description framework (rdf). http://www.w3.org/RDF/.

[5] Semantic web information management system (swims). http://semscape.cs.ucla.edu.

[6] Sparql query language for rdf.

[7] Stanford corenlp. http://nlp.stanford.edu/software/corenlp.shtml.

[8] Charniak nlp parser. ftp://ftp.cs.brown.edu/pub/nlparser/, 2008.

[9] R. Alur and P. Madhusudan. Adding nesting structure to words. In Developments in Language Theory, 2006.

[10] M. Atzori and C. Zaniolo. Swipe: searching wikipedia by example. In $W W W$, pages 309-312, 2012.

[11] E. Charniak and M. Elsner. Em works for pronoun anaphora resolution. In $E A C L$, pages 148-156, 2009.

[12] S. A. Cook. The complexity of theorem-proving procedures. In STOC, pages $151-158,1971$.

[13] H. Cunningham, D. Maynard, K. Bontcheva, and V. Tablan. Gate: an architecture for development of robust hlt applications. In Recent Advanced in Language Processing, pages 168-175, 2002.

[14] B. J. Grosz, S. Weinstein, and A. K. Joshi. Centering: a framework for modeling the local coherence of discourse. Comput. Linguist., 21(2):203-225, June 1995.

[15] A. Haghighi and D. Klein. Unsupervised coreference resolution in a nonparametric bayesian model. In ACL, 2007.

[16] A. Haghighi and D. Klein. Simple coreference resolution with rich syntactic and semantic features. In EMNLP, 2009.

[17] R. Hahn, C. Bizer, C. Sahnwaldt, C. Herta, S. Robinson, M. Bürgle, H. Düwiger, and U. Scheel. Faceted wikipedia search. In BIS, 2010.

[18] J. Hobbs. Resolving pronoun references. Lingua, 44:311-338, 1978.

[19] A. Kehler, D. Appelt, L. Taylor, and A. Simma. Competitive self-trained pronoun interpretation. In HLT-NAACL, pages 33-36, 2004.

[20] D. Kerr, H. Mousavi, and M. Iseli. Automatic short essay scoring using natural language processing to extract semantic information in the form of propositions. In (CRESST Report 831). UCLA, 2013.

[21] K. Kipper, A. Korhonen, N. Ryant, and M. Palmer. A large-scale classification of English verbs. JLRE, 42(1):21-40-40, Mar. 2008.

[22] D. Klein and C. D. Manning. Accurate unlexicalized parsing. In $A C L$, pages 423-430, 2003.

[23] S. Lappin and H. J. Leass. An algorithm for pronominal anaphora resolution. Comput. Linguist., 20(4):535-561, 1994.

[24] T. Lee, Z. Wang, H. Wang, and S. won Hwang. Web scale taxonomy cleansing. PVLDB, 4(12):1295-1306, 2011.

[25] M. D. Marneffe, B. Maccartney, and C. D. Manning. Generating typed dependency parses from phrase structure parses. In LREC, 2006.

[26] O. Medelyan, D. N. Milne, C. Legg, and I. H. Witten. Mining meaning from wikipedia. Int. J. Hum.-Comput. Stud., 67(9):716-754, 2009.

[27] H. Mousavi, S. Gao, and C. Zaniolo. Discovering attribute and entity synonyms for knowledge integration and semantic web search. SSW, 2013.

[28] H. Mousavi, S. Gao, and C. Zaniolo. Ibminer: A text mining tool for constructing and populating infobox databases and knowledge bases. $P V L D B, 6(12): 1330-1333,2013$.

[29] H. Mousavi, D. Kerr, M. Iseli, and C. Zaniolo. Deducing infoboxes from unstructured text in wikipedia pages. In CSD Technical Report \#130001, UCLA, 2013.

[30] H. Mousavi, D. Kerr, M. Iseli, and C. Zaniolo. Ontoharvester: An unsupervised ontology generator from free text. In CSD Technical Report \#130003, UCLA, 2013.

[31] D. Nadeau and S. Sekine. A survey of named entity recognition and classification. Lingvisticae Investigationes, 30(1):3-26, Jan. 2007.

[32] J. D. Novak and G. D. B. Learning how to learn. Cambridge University Press, N.Y., 1984.

[33] H. Poon and P. Domingos. Joint unsupervised coreference resolution with markov logic. In $E M N L P, 2008$.

[34] K. Raghunathan, H. Lee, S. Rangarajan, N. Chambers, M. Surdeanu, D. Jurafsky, and C. D. Manning. A multi-pass sieve for coreference resolution. In EMNLP, 2010.

[35] J. F. Sowa. Conceptual graphs for a data base interface. IBM Journal of Research and Development, 20(4):336-357, 1976.

[36] M. M. Stark and R. F. Riesenfeld. Wordnet: An electronic lexical database. In Eurographics Workshop on Rendering. MIT Press, 1998.

[37] A. Tan. Text mining: The state of the art and the challenges. In PAKDD, pages $65-70,1999$. 